\title{
SÍNDROME DE GORHAM-STOUT: "DOENÇA DO OSSO FANTASMA"
}

\section{GORHAM-STOUT SYNDROME: "PHANTOM BONE DISEASE"}

\author{
Gabriel El-Kouba', Romilton de Araújo Santos ${ }^{1}$, Paulo César Pilluski² ${ }^{2}$ Antonio Severo ${ }^{3}$, Osvandré Lech ${ }^{4}$
}

\section{RESUMO}

A síndrome de Gorham-Stout é uma doença que apresenta osteólise idiopática de um osso ou área contígua próxima. A etiologia é desconhecida, sendo uma condição rara, de difícil diagnóstico e tratamento controverso. Acomete pessoas sem distinção quanto à idade e ao sexo. Neste trabalho realizamos uma revisão bibliográfica da doença, dando enfoque específico no diagnóstico diferencial, e demonstramos o acompanhamento de um paciente com esta síndrome, desde o seu diagnóstico, tratamento e estado atual de evolução.

Descritores - Osteólise Essencial/etiologia; Osteólise/diagnóstico; Osteólise/radiografia; Clavícula

\section{ABSTRACT}

Gorham-stout syndrome is a disease that presents idiopathic osteolysis of a bone or contiguous area. The etiology is unknown. It is a rare condition, difficult to diagnose and with controversial treatment. This condition affects persons with no distinction as to age or sex. In this study, we conduct a bibliographic review of the disease, specifically focusing on differential diagnosis, and follow a patient with this syndrome from the time of its diagnosis, through treatment, to its current state of evolution.

Keywords - Osteolysis Essential/etiology; Osteolysis Essential/ diagnosis; Osteolysis Essential/radiography; Clavicle

\section{INTRODUÇÃO}

O termo osteólise indica redução na quantidade de tecido ósseo visualizada em uma radiografia. Essa condição pode ser causada por grande número de lesões conhecidas, porém existem poucos casos de osteólise idiopática.

Em 1838, Jackson ${ }^{(1)}$ descreveu a osteólise completa do úmero de um menino de 12 anos. Em 1955, Gorham e Stout ${ }^{(2)}$ relataram as principais características patológicas daquela que foi denominada de "doença do osso desaparecido com alterações vasculares intraósseas”. Desde então essa doença vem sendo chamada de síndrome de Gorham, síndrome de Gorham-Stout, osteólise maciça, osteólise idiopática, doença do osso desaparecido, doença do osso fantasma, absorção espontânea do osso, atrofia progressiva do osso ou hemangiomatose e linfangiomatose do osso.
Foram descritos menos de 200 casos na literatura ${ }^{(3)}$.

A síndrome de Gorham-Stout se apresenta com osteólise de um osso ou área óssea contígua próxima do foco, sem respeitar limites articulares. Pode acometer qualquer porção do esqueleto, mas é monocêntrica. As estruturas mais envolvidas são os ossos do crânio e das cinturas escapular e pélvica ${ }^{(2,4-6)}$. É doença rara, de etiologia desconhecida, em que a osteólise inicia pela proliferação de estruturas vasculares originadas no tecido ósseo causando a destruição da matriz óssea(7).

Essa condição pode se apresentar em qualquer idade, mas geralmente é reconhecida em crianças e adultos jovens. Não há predileção por sexo ou padrão de herança genética $^{(8,9)} \mathrm{e}$, até então, não houve nenhum caso com história familiar ${ }^{(4)}$. Sabe-se que em $57 \%$ dos casos existe uma história de trauma associado ${ }^{(7)}$.

\footnotetext{
1 - Residente do Serviço de Cirurgia do Ombro e Cotovelo do Instituto de Ortopedia e Traumatologia de Passo Fundo, RS.

2 - Preceptor do Serviço de Residência Médica do Instituto de Ortopedia e Traumatologia de Passo Fundo, RS.

3 - Instrutor do Serviço de Cirurgia da Mão do Instituto de Ortopedia e Traumatologia de Passo Fundo, RS.

4 - Chefe do Serviço de Residência Médica do Instituto de Ortopedia e Traumatologia de Passo Fundo, RS.

Trabalho realizado no Instituto de Ortopedia e Traumatologia (IOT) - Passo Fundo, RS.

Correspondência: IOT - Instituto de Ortopedia e Traumatologia de Passo Fundo. Rua Uruguai, 2050 99010-112 - Passo Fundo, RS.

E-mail: ensino@oitrs.com.br; ombromao@iotrs.com.br
}

Trabalho recebido para publicação 13/08/09 aceito para publicação 18/10/10. 
Com relação ao quadro clínico, não apresenta grande sintomatologia, sendo a aparência do quadro radiológico muito pior do que o quadro clínico. Durante a fase aguda o paciente se queixa de dor, edema, deformidade progressiva e contraturas. Apesar disto, a função do membro acometido permanece marcadamente boa. Exames laboratoriais geralmente são normais e auxiliam o médico na exclusão de outros diagnósticos, sendo que a fosfatase alcalina pode ser elevada quando o paciente apresenta fraturas $^{(10)}$.

O envolvimento costuma ser contíguo, não ocorrendo múltiplos focos ou metástases. Existe apenas um caso de degeneração maligna documentada ocorrida em um paciente com o diagnóstico de hipernefroma ${ }^{(11)}$.

As mortes causadas pela doença ocorreram em pacientes nos quais o processo estava localizado nos arcos costais, mandíbula ou corpos vertebrais, levando a complicações respiratórias fatais, obstrução de vias aéreas ou compressão da medula espinhal ${ }^{(4,12,13)}$. É comum ocorrer parada da osteólise de forma espontânea após anos de destruição óssea ${ }^{(2)}$. O resultado final é deformidade e impotência funcional.

Quilotórax é uma complicação rara, porém é grave e está associada com osteólise da cintura escapular ou da coluna torácica ${ }^{(9)}$. Resulta do acometimento do ducto torácico, ou comunicação da displasia linfática com a cavidade pleural. É frequentemente fatal e cursa com linfopenia, desnutrição e infecções oportunistas superpostas ${ }^{(14)}$.

Nas radiografias dos ossos longos o fenômeno começa com focos de radioluscência intramedular e subcortical, parecendo quadro de osteoporose desigual. Tem progressão lenta e irregular com redução concêntrica da diáfise dos ossos, afilando as extremidades envolvidas e causando o desaparecimento completo do osso, exceto se ocorrer remissão espontânea ${ }^{(4-6,10,12)}$. Fraturas patológicas podem acontecer e raramente consolidam. Assim o processo osteolítico continua através dos fragmentos ${ }^{(4,15,16)}$. Um fato típico e notável é a ausência de esclerose ou reação osteoblástica.

A tomografia axial computadorizada (TAC) é útil para delimitar a extensão dos tecidos moles, além de possibilitar a biópsia guiada. A TAC com reconstrução tridimensional (3D) auxilia no planejamento cirúrgico para reconstruções $^{(17)}$.

A linfangiografia pode ser utilizada para avaliar o comprometimento do ducto torácico em pacientes com quilotórax. Os vasos linfáticos e linfonodos têm aparência normal, apesar do fluxo linfático alterado poder levar a obstrução e ao edema ${ }^{(18)}$.
A angiografia retrata a ausência de neovascularização na área envolvida e a cintilografia evidencia captação normal em todo o esqueleto ou aumento da captação nas áreas envolvidas ${ }^{(18,19)}$.

A ressonância nuclear magnética (RNM) demonstra desaparecimento morfológico do osso e áreas com intensidade de sinal aumentadas ou diminuídas, que representam hemorragias em estágios diferentes ${ }^{(18)}$.

O diagnóstico diferencial da síndrome de Gorham inclui hemangioma ósseo, angiossarcoma, osteólise essencial e osteólise hereditária. O padrão radiográfico é muito semelhante, mas nestas doenças não ocorrem grandes derrames pleurais e demais alterações pulmonares ${ }^{(3)}$.

Os hemangiomas esqueléticos podem causar osteólise e apresentam características histológicas muito similares à síndrome de Gorham. No entanto, os hemangiomas têm crescimento limitado com tendência a preservar estoque ósseo cortical, e não apresentam disseminação para as partes moles adjacentes ${ }^{(3)}$.

Os angiossarcomas, mesmo quando bem diferenciados, apresentam atipia celular focal e células endoteliais com tendência à proliferação. Já na síndrome de Gorham os vasos apresentam camada única de células endoteliais $^{(1,7)}$.

A osteólise essencial causa reabsorção óssea carpal e/ou tarsal, cursando com falência renal progressiva e sem alterações vasculares. Já a osteólise hereditária ocorre na infância e se apresenta principalmente nas mãos e pés, sem cursar com alterações vasculares da mesma forma ${ }^{(3)}$. Portanto, através da multicentricidade destas doenças, podemos realizar o diagnóstico diferencial com a doença descrita. Outras causas possíveis de osteólise advêm de doenças sistêmicas como artrite reumatoide, sífilis, hiperparatireoidismo, mieloma múltiplo e linfoma. No entanto, a avaliação histológica difere das alterações encontradas nos pacientes com a síndrome de Gorham ${ }^{(7)}$.

O tratamento da síndrome de Gorham deve ser instituído quando o processo é progressivo e a osteólise é extensa. Baseia-se na ressecção local, com ou sem protetização, radioterapia ou amputação. A ressecção incompleta da lesão raramente é curativa, e o processo de lise óssea se perpetua ${ }^{(20,21)}$. A imobilização não altera o prognóstico, assim como administração de estrogênio, androgênio, magnésio, cálcio, extratos adrenérgicos, vitamina $\mathrm{D}$, solução de alumínio, radiação ultravioleta, somatotrofina, extratos placentários, vitamina B12, aminoácidos ou transfusão sanguínea de crianças em crescimento $^{(22-24)}$. 
Devido à raridade da doença, descrevemos a seguir o relato de um caso de paciente portador da síndrome de Gorham-Stout.

\section{RELATO DE CASO}

Paciente, masculino, com data de nascimento 20/08/1996, atendido pela primeira vez pelo autor sênior (OL) aos 12 anos, com história de acompanhamento médico desde os seis anos. Sem alterações na infância precoce ou doenças prévias. Em 2002, aos seis anos, teve quadro de queda de própria altura com fratura de clavícula esquerda. O tratamento foi realizado por ortopedista, de forma conservadora através de imobilização com faixa em "oito posterior" e relatos de consolidação óssea com formação de “calo ósseo”.

Após um ano teve outra queda de própria altura com nova fratura da clavícula, próximo ao sítio de consolidação da fratura anterior. Foi realizado novo tratamento conservador com imobilização em tipoia e consolidação da fratura.

Em 2004, então com oito anos, teve trauma direto causado por trauma direto sobre o ombro esquerdo, resultando em nova fratura de clavícula e tratamento semelhante aos anteriores. Durante consulta após três semanas da terceira fratura da clavícula, as radiografias evidenciaram "desaparecimento” de parte da clavícula esquerda (Figura 1). A equipe médica que acompanhava o caso realizou radiografia de crânio, bacia e coluna, que se mostraram normais. Devido a suspeita de doença maligna, a equipe completou o estadiamento da lesão solicitando TAC, RNM e biópsia. A ressonância

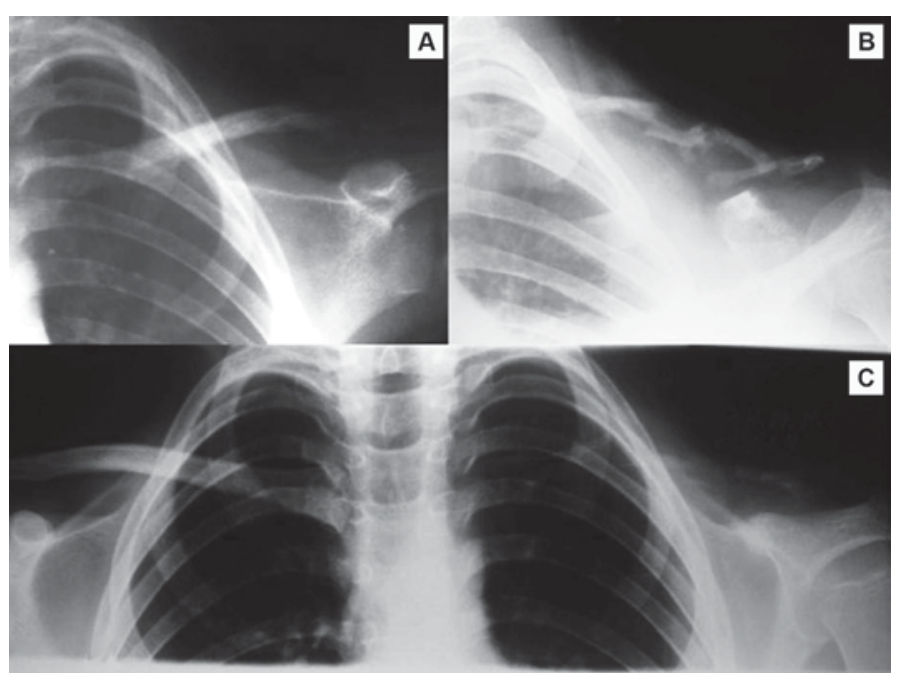

Figura 1 - Evolução da osteólise da clavícula esquerda causada pela doença de Gorham-Stout. Radiografias da clavícula em fevereiro de 2004 (A), em setembro de 2004 (B) e em janeiro de 2005 (C), mostrando a rápida evolução da osteólise. demonstrou não haver comprometimento das partes moles, e a biópsia resultou em alterações histopatológicas sugestivas de "angioma ósseo”.

Através da história do paciente e dos resultados dos exames, chegou-se ao diagnóstico da doença de GorhamStout. A partir de então foi proposto seguimento anual através da avaliação pelo exame físico e radiografias. Quanto ao tratamento, o paciente foi estimulado a reforçar a musculatura do membro superior esquerdo através de aulas de natação.

O seguimento foi realizado durante três anos, até que em 2008, aos 12 anos, a avaliação radiológica evidenciou que o padrão de osteólise da clavícula havia se estabilizado, porém demonstrou lesões em região superior da escápula esquerda. TAC e RNM que confirmaram a presença de lesões osteolíticas na escápula até o maciço glenoideo, coracoide e acrômio (Figura 2). A eletroneuromiografia foi normal. Clinicamente, o paciente foi apresentando impotência funcional com limitação do arco de movimento do ombro esquerdo, sem nenhuma alteração clínica de redução da ventilação pulmonar.

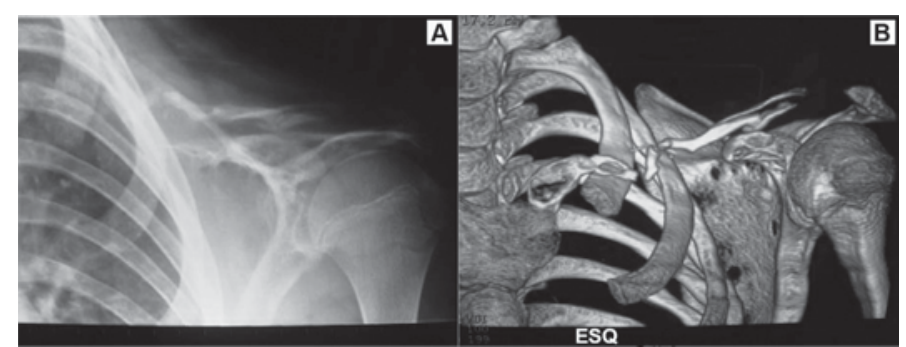

Figura 2 - Imagem radiográfica atual evidenciando o comprometimento escapular com focos de osteólise (A). Imagem de TAC com reconstrução digital em 3D exibindo a osteólise clavicular esquerda intensa e focos de comprometimento escapular associado (B).

No momento, a evolução da osteólise parece ter estacionado. O tratamento instituído neste momento é de fisioterapia e hidroterapia, focadas no reforço muscular dos estabilizadores da escápula, manguito rotador e deltoide, principalmente para manutenção do arco de movimento. O paciente segue em uso de terapia antirreabsortiva através da ingestão de bisfosfonado e calcitonina. As consultas para seguimento devem ser realizadas trimestralmente para observação e reavaliação do quadro. O quadro clínico do paciente pode ser evidenciado nas imagens clínicas abaixo (Figura 3).

\section{DISCUSSÃO}

A síndrome de Gorham-Stout deve ser diagnosticada com base na história clínica, imagens radiográficas e, principalmente, pelo conhecimento da doença. Den- 

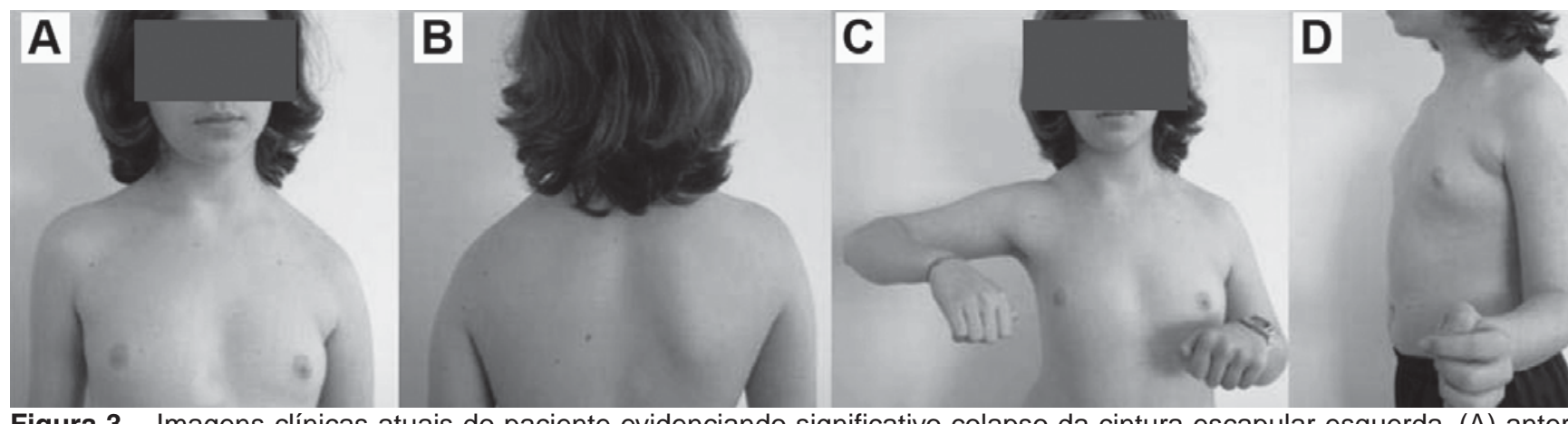

Figura 3 - Imagens clínicas atuais do paciente evidenciando significativo colapso da cintura escapular esquerda. (A) anterior em repouso, (B) posterior, $(C)$ incapacidade de abdução, (D) perfil esquerdo em rotação externa.

tre as várias possíveis causas de osteólise secundária, devemos diferenciar de atrofia difusa, algodistrofia, tumores primários e metastáticos, hiperparatireoidismo, gota, pseudartrose congênita, doenças granulomatosas, artrite reumatoide, diabetes mellitus, artrite psoriática, osteomielite, mastocistose sistêmica, necrose asséptica, artropatia neurogênica, terapia prolongada com corticoides, cisto ósseo aneurismático e hemangiomatose óssea ${ }^{(25)}$. As osteólises idiopáticas são um grupo heterogêneo de doenças raras. Hardegger et al ${ }^{(26)}$ propuseram uma classificação para estas osteólises composta de cinco tipos (Tabela 1).

Existiram várias publicações nas quais foram descritos pacientes que não se enquadraram em nenhum dos cinco subtipos descritos nesta classificação. White descreveu quatro pacientes com osteólise multifocal associadas com graves lesões de pele ${ }^{(27)}$. Beals e Bird ${ }^{(28)}$ observaram um caso de osteólise carpo tarsal sem nefropatia ou padrão hereditário.

O paciente descrito neste relato de caso teve quadro de osteólise monocêntrica iniciada durante a infância, sem história familiar de lesões osteolíticas ou nefropatias. Utilizando-se esta classificação, chegamos à conclusão de se tratar de um tipo 4 - síndrome de Gorham-Stout.

O mecanismo patogênico exato desta doença ainda não é conhecido. Existem controvérsias acerca da presença ou não de osteoclastos no foco de lesão. Spieth et al acreditam que a reabsorção é especificamente causada pelos osteoclastos ${ }^{(29)}$.

Existe grande variedade de explicações para a patogênese. Dentre elas, citamos a presença de um hamartoma silencioso que se ativa e inicia a reabsorção após pequeno traumatismo. Alguns autores acreditam que a angiomatose é a responsável por todo o quadro ${ }^{(2,11)}$. Outros descrevem alterações neurovasculares como as vistas na atrofia de Sudeck ${ }^{(30)}$. Thompson e Shurman ${ }^{(31)}$ sugerem que a doença é a aberração primária do tecido vascular ósseo associada com tecido de granulação. Young et $a l^{(32)}$ acreditavam que a osteólise é atribuída à displasia endotelial de linfáticos e vasos sanguíneos.

Desde que o osteoclasto é a única célula capaz de reabsorver osso, entende-se que a síndrome de GorhamStout pode representar patologicamente um desarranjo da

Tabela 1 - Classificação de osteólise idiopática de acordo com Hardegger et a/ ${ }^{(26)}$.

Tipos

\section{Osteólise multicêntrica hereditária com transmissão dominante}

2. Osteólise multicêntrica hereditária com transmissão recessiva

3. Osteólise multicêntrica não hereditária com nefropatia
Entre dois-sete anos, dor e edema espontâneos iniciam nas mãos e pés, com osteólise carpo tarsal. Progressão cessa na adolescência.

Similar ao tipo 1, mas pode estar associada a severa osteoporose generalizada.

Aparece na infância. Ocorre desaparecimento gradual dos ossos do carpo e tarso, porém em menor grau e associada com proteinúria. Morte ocorre por falência renal ou hipertensão maligna.

Ocorrência monocêntrica em qualquer parte do esqueleto, podendo iniciar em qualquer idade. Normalmente, encontra-se tecido hemangiomatoso nos focos de osteólise. Não é hereditário e não é associado à nefropatia. A osteólise costuma cessar após alguns anos.

Rara síndrome autossômica recessiva. Osteólise carpo tarsal associada a contraturas, redução da estatura, lesões de pele, alterações na córnea e osteoporose sem nefropatia.
5. Síndrome de Winchester 
atividade osteoclástica. Esses osteoclastos são estimulados por ação parácrina ou autócrina, reabsorvendo o tecido ósseo que posteriormente é trocado por tecido fibroso vascularizado. Portanto, a aparente contradição acerca da presença ou ausência destas células na doença pode ser explicada pelas diferentes fases da síndrome ${ }^{(25)}$.

Parece sensato realizar o tratamento dos pacientes portadores desta doença com terapia antirreabsortiva como bisfosfonados e calcitonina, que devem ser iniciados em fase precoce na tentativa de redução da progressão do processo osteolítico ${ }^{(25)}$. Porém, ainda faltam estudos comprovando estes dados devido à raridade da síndrome.

\section{REFERÊNCIAS}

1. Jackson JB. A boneless arm. Boston Med Surg J. 1838;18:368-9.

2. Gorham LW, Stout AP. Massive osteolysis (acute spontaneous absorption of bone, phantom bone, disappearing bone): its relation to hemangiomatosis. J Bone Joint Surg Am. 1955;37(5):985-1004.

3. Collins J. Case 92: Gorham syndrome. Radiology. 2006;238(3):1066-9.

4. Gowin W, Rahmanzadeh R. Radiologic diagnosis of massive idiopathic osteolysis (Gorham-Stout Syndrome). Rontgenpraxis. 1985;38(4):128-34.

5. Horst M, Zsernaviczky J, Delling G. A rare case of so-called idiopathic osteolysis associated with a lymphangioma of the fibula. Z Orthop Ihre Grenzgeb. 1979;117(1):88-95.

6. Flörchinger A, Böttger E, Claass-Böttger F, Georgi M, Harms J. Gorham Stout syndrome of the spine. Case report and review of the literature. Rofo. 1998;168(1):68-76.

7. Choma ND, Biscotti CV, Bauer TW, Mehta AC, Licata AA. Gorham's syndrome: a case report and review of the literature. Am J Med. 1987;83(6):1151-6.

8. Touraine R, Bernard JP, Trouillier JP, Balandreau AM. Chylothorax and Gorham's disease (or regional massive osteolysis). J Fr Med Chir Thorac. 1971;25(3):315-26.

9. Chavanis N, Chaffanjon P, Frey G, Vottero G, Brichon PY. Chylothorax complicating Gorham's disease. Ann Thorac Surg. 2001;72(3):937-9.

10. Johnson PM, McClure JG. Observations of massive osteolysis: a review of the literature and report of a case. Radiology. 1958;71(1):28-42.

11. Fretz CJ, Jungi WF, Neuweiler J, Haertel M. The malignant degeneration of Gorham-Stout disease? Rofo. 1991;155(6):579-81.

12. Tilling G, Skobowytsh B. Disappearing bone disease, morbus Gorham. Report of a case. Acta Orthop Scand. 1968;39(3):398-406.

13. Ellis DJ, Adams TO. Massive osteolysis: report of case. J Oral Surg. 1971;29(9):659-63.

14. Riantawan P, Tansupasawasdikul S, Subhannachart P. Bilateral chylothorax complicating massive osteolysis (Gorham's syndrome). Thorax. 1996;51(12):1277-8.

15. Gorham LW, Wright AW, Schultz HH, Maxon FC. Disappearing bones: a rare form of massive osteolysis: report of 2 cases, 1 with autopsy findings. Am J Med. 1954;17(5):674-82.

16. Kery L, Wouters HW. Massive osteolysis: report of two cases. J Bone Joint Surg Br. 1970;52(3):452-9.

17. Vinée P, Tanyü MO, Hauenstein KH, Sigmund G, Stöver B, Adler CP. CT and MRI of Gorham syndrome. J Comput Assist Tomogr. 1994;18(3):985-9.
Outra possibilidade terapêutica consiste na utilização da radioterapia. Carneiro e Steglich relataram um caso de paciente do sexo feminino com osteólise progressiva nos metacarpos, onde a estabilização da evolução por quatro meses após ciclo de 21 dias de radioterapia com 2.400 rads reforçou a ideia de enxertia óssea nos focos da osteólise ${ }^{(33)}$. No entanto, a doença manteve a progressão e reabsorveu todo o enxerto utilizado. O caso descrito por estes autores teve parada de sua progressão. Nos casos graves, com complicações pulmonares ou neurológicas está indicado o tratamento cirúrgico para ressecção do tecido doente na tentativa de cessar a progressão da doença.
18. Dominguez R, Washowich TL. Gorham's disease or vanishing bone disease: plain film CT, and MRI findings of two cases. Pediatr Radiol. 1994;24(5):316-8.

19. Tie ML, Poland GA, Rosenow EC 3rd. Chylothorax in Gorham's syndrome: a common complication of a rare disease. Chest. 1994;105(1):208-13.

20. Chambers TJ. The cellular basis of bone resorption. Clin Orthop Relat Res. 1980;(151):283-93.

21. Poirier $\mathrm{H}$. Massive osteolysis of the humerus treated by massive resection and prosthetic replacement. J Bone Joint Surg Br. 1968;50(1):158-60.

22. Butler RW, McCance RA, Barrett AM. Unexplained destruction of the shaft of the femur in a child. J Bone Joint Surg Br. 1958;40(1):487-93.

23. Phillips RM, Bush OB Jr, Hall HD. Massive osteolysis (phantom bone, disappearing bone). Report of a case with mandibular involvement. Oral Surg Oral Med Oral Pathol. 1972;34(6):886-96.

24. Ross JL, Schinella R, Shenkman L. Massive osteolysis. An unusual cause of bone destruction. Am J Med. 1978;65(2):367-72.

25. Möller G, Priemel M, Amling M, Werner M, Kuhlmey AS, Delling G. The GorhamStout syndrome (Gorham's massive osteolysis). A report of six cases with histopathological findings. J Bone Joint Surg Br. 1999;81(3):501-6.

26. Hardegger F, Simpson LA, Segmueller G. The syndrome of idiopathic osteolysis: classification, review and case report. J Bone Joint Surg Br. 1985;67(1):89-93.

27. White AA. Disappearing bone disease with arthropathy and severe scarring of the skin. A report of four cases seen in South Vietnam. J Bone Joint Surg Br. 1971;53(2):303-9.

28. Beals RK, Bird CB. Carpal and tarsal osteolysis. A case report and review of the literature. J Bone Joint Surg Am. 1975;57(5):681-6.

29. Spieth ME, Greenspan A, Forrester DM, Ansari AN, Kimura RL, Gleason-Jordan I. Gorham's disease of the radius: radiographic, scintigraphic, and MRI findings with pathologic correlation. A case report and review of the literature. Skeletal Radiol. 1997;26(11):659-63.

30. Knoch HG. Die Gorhamsche Krankheit aus klinischer Sicht. Zentralbl Chir. 1963;18:674-83.

31. Thompson JS, Schurman DJ. Massive osteolysis: case report and review of the literature. Clin Orthop Relat Res. 1974;(103):206-11.

32. Young JW, Galbraith M, Cunningham J, Roof BS, Vujic I, Gobien RP et al. Progressive vertebral collapse in diffuse angiomatosis. Metab Bone Dis Relat Res. 1983;5(2):53-60.

33. Carneiro RS, Steglich V. "Disappearing bone disease" in the hand. J Hand Surg Am. 1987;12(4):629-34. 